\title{
Kernel Entropy Component Analysis Pre-images for Pattern Denoising
}

\author{
Robert Jenssen and Ola Storås \\ Department of Physics and Technology, University of Troms $\varnothing$, 9037 Troms $\varnothing$, Norway \\ Tel.: (+47) 776-46493; Fax: (+47) 776-45580 \\ robert.jenssen@uit.no
}

\begin{abstract}
The recently proposed kernel entropy component analysis (kernel ECA) technique may produce strikingly different spectral data sets than kernel PCA for a wide range of kernel sizes. In this paper, we investigate the use of kernel ECA as a component in a denoising technique previously developed for kernel PCA. The method is based on mapping noisy data to a kernel feature space, for then to denoise by projecting onto a kernel ECA subspace. The denoised data in the input space is obtained by computing pre-images of kernel ECA denoised patterns. The denoising results are in several cases improved.
\end{abstract}

\section{Introduction}

Kernel entropy component analysis was proposed in [1] $]^{1}$. The idea is to represent the input space data set by a projection onto a kernel feature subspace spanned by the $k$ kernel principal axes which corresponds to the largest contributions of Renyi entropy with regard to the input space data set. This mapping may produce a radically different kernel feature space data set than kernel PCA, depending on the kernel size used.

Recently, kernel PCA 2] has been used for denoising by mapping a noisy input space data point into a Mercer kernel feature space, for then to project the data point onto the leading kernel principal axes obtained using kernel PCA based on clean training data. This is the actual denoising. In order to represent the input space denoised pattern, i.e. the pre-image of the kernel feature space denoised pattern, a method for finding the pre-image is needed. Mika et al. 3] proposed such a method using an iterative scheme. More recently, Kwok and Tsang [4] proposed an algebraic method for finding the pre-image, and reported positive results compared to [3]. This method has also been used in [5].

In this paper, we introduce kernel ECA for pattern denoising. Clean training data is used to obtain the "entropy subspace" in the kernel feature space. A noisy input pattern is mapped to kernel space and then projected onto this subspace. This removes the noise in a different manner as opposed to using kernel PCA

${ }^{1}$ In [1, this method was referred to as kernel maximum entropy data transformation. However, kernel entropy component analysis (kernel ECA) is a more proper name, and will be used subsequently. 
for this purpose. Subsequently, Kwok and Tsang's 4 method for finding the pre-image, i.e. the denoised input space pattern, is employed. Positive results are obtained.

This paper is organized as follows. In Section 2, we review the kernel ECA method, and in Section 3, we explain how to use kernel ECA for denoising in combination with Kwok and Tsang's 4 pre-image method. We report experimental results in Section 4 and conclude the paper with Section 5 .

\section{Kernel Entropy Component Analysis}

We first discuss how to perform kernel ECA based on a sample of data points. This is referred to as in-sample kernel ECA. Thereafter, we discuss how to project an out-of-sample data point onto the kernel ECA principal axes.

\section{$2.1 \quad$ In-Sample Kernel ECA}

The Renyi (quadratic) entropy is given by $H(p)=-\log V(p)$, where $V(p)=$ $\int p^{2}(\mathbf{x}) d \mathbf{x}$ and $p(\mathbf{x})$ is the probability density function generating the input space data set, or sample, $\mathcal{D}=\mathbf{x}_{1}, \ldots, \mathbf{x}_{N}$. By incorporating a Parzen window density estimator $\hat{p}(\mathbf{x})=\frac{1}{N} \sum_{\mathbf{x}_{t} \in \mathcal{D}} k_{\sigma}\left(\mathbf{x}, \mathbf{x}_{t}\right)$, [1] showed that an estimator for the Renyi entropy is given by

$$
\hat{V}(p)=\frac{1}{N^{2}} \mathbf{1}^{T} \mathbf{K} \mathbf{1},
$$

where element $\left(t, t^{\prime}\right)$ of the kernel matrix $\mathbf{K}$ equals $k_{\sigma}\left(\mathbf{x}_{t}, \mathbf{x}_{t^{\prime}}\right)$. The parameter $\sigma$ governs the width of the window function. If the Parzen window is positive semidefinite, such as for example the Gaussian function, then a direct link to Mercer kernel methods is made (see for example [6]). In that case $\hat{V}(p)=\|\mathbf{m}\|^{2}$, where $\mathbf{m}=\frac{1}{N} \sum_{\mathbf{x}_{t} \in \mathcal{D}} \phi\left(\mathbf{x}_{t}\right)$ and $\phi\left(\mathbf{x}_{1}\right), \ldots, \phi\left(\mathbf{x}_{N}\right)$ represents the input data mapped to a Mercer kernel feature space. Note that centering of the kernel matrix does not make sense when estimating Renyi entropy. Centering means that $\mathbf{m}=\mathbf{0}$, which again results in $\hat{V}(p)=0$. Therefore, the kernel matrix is not centered in kernel ECA.

The kernel matrix may be eigendecomposed as $\mathbf{K}=\mathbf{E} \mathbf{D E} \mathbf{E}^{T}$, where $\mathbf{D}$ is a diagonal matrix storing the eigenvalues $\lambda_{1}, \ldots, \lambda_{N}$ and $\mathbf{E}$ is a matrix with the corresponding eigenvectors $\mathbf{e}_{1}, \ldots, \mathbf{e}_{N}$ as columns. Re-writing Eq. (1), we then have

$$
\hat{V}(p)=\frac{1}{N^{2}} \sum_{i=1}^{N}\left(\sqrt{\lambda_{i}} \mathbf{e}_{i}^{T} \mathbf{1}\right)^{2},
$$

where $\mathbf{1}$ is a $(N \times 1)$ ones-vector and $\sqrt{\lambda_{1}} \mathbf{e}_{1}^{T} \mathbf{1} \geq, \ldots, \geq \sqrt{\lambda_{N}} \mathbf{e}_{N}^{T} \mathbf{1}$.

Let the kernel feature space data set be represented by $\boldsymbol{\Phi}=\phi\left(\mathbf{x}_{1}\right), \ldots, \phi\left(\mathbf{x}_{N}\right)$. As shown for example in [7, the projection of $\boldsymbol{\Phi}$ onto the $i$ th principal axis $\mathbf{u}_{i}$ in kernel feature space defined by $\mathbf{K}$ is given by $P_{\mathbf{u}_{i}} \boldsymbol{\Phi}=\sqrt{\lambda_{i}} \mathbf{e}_{i}^{T}$. This reveals an interesting property of the Renyi entropy estimator. The $i$ th term in Eq. (2) in fact corresponds to the squared sum of the projection onto the $i$ th principal axis in kernel feature space. The first terms of the sum, i.e. the largest values, 
will contribute most to the entropy of the input space data set. Note that each term depends both on an eigenvalue and on the corresponding eigenvector.

Kernel entropy component analysis represents the input space data set by a projection onto a kernel feature subspace $U_{k}$ spanned by the $k$ principal axes corresponding to the largest "entropy components", that is, the eigenvalues and eigenvectors comprising the first $k$ terms in Eq. (2). If we collect the chosen $k$ eigenvalues in a $(k \times k)$ diagonal matrix $\mathbf{D}_{k}$ and the corresponding eigenvectors in the $(N \times k)$ matrix $\mathbf{E}_{k}$, then the kernel ECA data set is given by

$$
\boldsymbol{\Phi}_{e c a}=P_{U_{k}} \boldsymbol{\Phi}=\mathbf{D}_{k}^{\frac{1}{2}} \mathbf{E}_{k}^{T}
$$

The $i$ th column of $\boldsymbol{\Phi}_{\text {eca }}$ now represents $\boldsymbol{\Phi}\left(\mathbf{x}_{i}\right)$ projected onto the subspace. We refer to this as in-sample kernel ECA, since $\boldsymbol{\Phi}_{\text {eca }}$ represents each data point in the original input space sample data set $\mathcal{D}$. We may refer to $\boldsymbol{\Phi}_{\text {eca }}$ as a spectral data set, since it is composed of the eigenvalues (spectrum) and eigenvectors of the kernel matrix. The value of $k$ is a user-specified parameter. For an input data set which is composed of subgroups (as revealed by training data), 1] discusses how kernel ECA approximates the "ideal" situation by selecting the value of $k$ equal to the number of subgroups.

In contrast, kernel principal component analysis projects onto the leading principal axes, as determined solely by the largest eigenvalues of the kernel matrix. The kernel matrix may be centered or non-centered 2 . We denote the kernel matrix used in kernel PCA $\mathbf{K}^{\prime}=\mathbf{V} \boldsymbol{\Delta} \mathbf{V}^{T}$. The kernel PCA mapping is given by $\boldsymbol{\Phi}_{p c a}=\boldsymbol{\Delta}_{k}^{\frac{1}{2}} \mathbf{V}_{k}^{T}$, using the $k$ largest eigenvalues of $\mathbf{K}^{\prime}$ and the corresponding eigenvectors.

\subsection{Out-of-Sample Kernel ECA}

In a similar manner as in kernel PCA, out-of-sample data points may also be projected into the kernel ECA subspace obtained based on the sample $\mathcal{D}$. Let the out-of-sample data point be denoted by $\mathbf{x} \rightarrow \phi(\mathbf{x})$. The principal axis $\mathbf{u}_{i}$ in the kernel feature space defined by $\mathbf{K}$ is given by $\mathbf{u}_{i}=\frac{1}{\sqrt{\lambda_{i}}} \boldsymbol{\phi} \mathbf{e}_{i}$, where $\boldsymbol{\phi}=$ $\left[\phi\left(\mathbf{x}_{1}\right), \ldots, \phi\left(\mathbf{x}_{n}\right)\right]$ [7] . Moreover, the projection of $\phi(\mathbf{x})$ onto the direction $\mathbf{u}_{i}$ is given by

$$
P_{\mathbf{u}_{i}} \phi(\mathbf{x})=\mathbf{u}_{i}^{T} \phi(\mathbf{x})=\frac{1}{\sqrt{\lambda_{i}}} \mathbf{e}_{i}^{T} \mathbf{k}_{\mathbf{x}}
$$

where $\mathbf{k}_{\mathbf{x}}=\left[k_{\sigma}\left(\mathbf{x}, \mathbf{x}_{1}\right), \ldots, k_{\sigma}\left(\mathbf{x}, \mathbf{x}_{N}\right)\right]^{T}$. The projection $P_{U_{k}} \phi(\mathbf{x})$ of $\phi(\mathbf{x})$ onto the subspace $U_{k}$ spanned by the $k$ principal axes as determined by kernel ECA is then

$$
P_{U_{k}} \phi(\mathbf{x})=\sum_{i=1}^{k} P_{\mathbf{u}_{i}} \phi(\mathbf{x}) \mathbf{u}_{i}=\sum_{i=1}^{k} \frac{1}{\sqrt{\lambda_{i}}} \mathbf{e}_{i}^{T} \mathbf{k}_{\mathbf{x}} \frac{1}{\sqrt{\lambda_{i}}} \phi \mathbf{e}_{i}=\phi \mathbf{M} \mathbf{k}_{\mathbf{x}}
$$

\footnotetext{
${ }^{2}$ Most researchers center the kernel matrix in kernel PCA. But 7] shows that centering is not really necessary. In this paper we consider both.
} 
where $\mathbf{M}=\sum_{i=1}^{k} \frac{1}{\lambda_{i}} \mathbf{e}_{i} \mathbf{e}_{i}^{T}$ is symmetric. If using kernel PCA, then $\mathbf{D}_{k}^{\frac{1}{2}}$ and $\mathbf{E}_{k}$ is replaced by $\boldsymbol{\Delta}_{k}^{\frac{1}{2}}$ and $\mathbf{V}_{k}^{T}$ and $\mathbf{M}$ is adjusted accordingly. See [4] a detailed analysis of centered kernel PCA.

\section{Denoising and Pre-image Mapping}

Kernel ECA may produce a strikingly different spectral data set than kernel PCA, as will be illustrated in next section. We want to take advantage of this property for denoising. Given clean training data, the kernel ECA subspace $U_{k}$ may be found. When utilizing kernel ECA for denoising, a noisy out-of-sample data point $\mathbf{x}$ is projected onto $U_{k}$, resulting in $P_{U_{k}} \phi(\mathbf{x})$. If the subspace $U_{k}$ represents the clean data appropriately, this operation will remove the noise. The final step is the computation of the pre-image $\hat{\mathbf{x}}$ of $P_{U_{k}} \phi(\mathbf{x})$, yielding the input space denoised pattern. Here, we will adopt Kwok and Tsang's 4 method for finding the pre-image. The method presented in 4 assumes that the pre-image lies in the span of its $n$ nearest neighbors. The nearest neighbors of $\hat{\mathbf{x}}$ will be equal to the kernel feature space nearest neighbors of $P_{U_{k}} \phi(\mathbf{x})$, which we denote $\phi\left(\mathbf{x}_{n}\right) \in \mathcal{D}_{n}$. The algebraic method for finding the pre-image needs Euclidean distance constraints between $\hat{\mathbf{x}}$ and the neighbors $\mathbf{x}_{n} \in \mathcal{D}_{n}$. Kwok and Tsang [4] show how to obtain these constraints in kernel PCA via Euclidean distances in the kernel feature space, using an invertible kernel such as the Gaussian. In the following, we show how to obtain the relevant kernel ECA Euclidean distances. We use a Gaussian kernel function. The pseudo-code for kernel ECA pattern denoising is summarized as

\section{Pseudo-Code of Kernel ECA Pattern Denoising}

- Based on noise free training data $\mathbf{x}_{1}, \ldots, \mathbf{x}_{N}$ determine $\mathbf{K}$ and the kernel ECA projection $\boldsymbol{\Phi}_{e c a}=\mathbf{D}_{k}^{\frac{1}{2}} \mathbf{E}_{k}^{T}$ onto the subspace $U_{k}$.

- For a noisy pattern $\mathbf{x}$ do

1. Project $\phi(\mathbf{x})$ onto $U_{k}$ by $P_{U_{k}} \phi(\mathbf{x})$

2. Determine the feature space Euclidean distances from $P_{U_{k}} \phi(\mathbf{x})$ to its $n$ nearest neighbors $\phi\left(\mathbf{x}_{n}\right) \in \mathcal{D}_{n}$

3. Translate the feature space Euclidean distances into input space Euclidean distances

4. Find the pre-image $\hat{\mathbf{x}}$ using the input space Euclidean distances (Kwok and Tsang [4])

\subsection{Euclidean Distances Based on Kernel ECA}

We need the Euclidean distances between $P_{U_{k}} \phi(\mathbf{x})$ and $\phi\left(\mathbf{x}_{n}\right) \in \mathcal{D}_{n}$. These are obtained by

$$
\tilde{d}^{2}\left[P_{U_{k}} \phi(\mathbf{x}), \phi\left(\mathbf{x}_{n}\right)\right]=\left\|P_{U_{k}} \phi(\mathbf{x})\right\|^{2}+\left\|\phi\left(\mathbf{x}_{n}\right)\right\|^{2}-2 P_{U_{k}}^{T} \phi(\mathbf{x}) \phi\left(\mathbf{x}_{n}\right),
$$


where $\left\|\phi\left(\mathbf{x}_{n}\right)\right\|^{2}=K_{n n}=k_{\sigma}\left(\mathbf{x}_{n}, \mathbf{x}_{n}\right)$. Based on the discussion in 2.2, we have

$$
\left\|P_{U_{k}} \phi(\mathbf{x})\right\|^{2}=\left(\phi \mathbf{M} \mathbf{k}_{\mathbf{x}}\right)^{T}\left(\phi \mathbf{M} \mathbf{k}_{\mathbf{x}}\right)=\mathbf{k}_{\mathbf{x}}^{T} \mathbf{M K M k}_{\mathbf{x}}
$$

since $\mathbf{M}^{T}=\mathbf{M}$ and $\boldsymbol{\phi}^{T} \boldsymbol{\phi}=\mathbf{K}$. Moreover

$$
P_{U_{k}}^{T} \phi(\mathbf{x}) \phi\left(\mathbf{x}_{n}\right)=\left(\phi \mathbf{M} \mathbf{k}_{\mathbf{x}}\right)^{T} \phi\left(\mathbf{x}_{n}\right)=\mathbf{k}_{\mathbf{x}}^{T} \mathbf{M} \phi^{T} \phi\left(\mathbf{x}_{n}\right)=\mathbf{k}_{\mathbf{x}}^{T} \mathbf{M} \mathbf{k}_{\mathbf{x}_{n}},
$$

where $\phi^{T} \phi\left(\mathbf{x}_{n}\right)=\mathbf{k}_{\mathbf{x}_{n}}=\left[k_{\sigma}\left(\mathbf{x}_{n}, \mathbf{x}_{1}\right), \ldots, k_{\sigma}\left(\mathbf{x}_{n}, \mathbf{x}_{N}\right)\right]^{T}$. Hence, we obtain a formula for finding the Euclidean distance as $\tilde{d}^{2}\left[P_{U_{k}} \phi(\mathbf{x}), \phi\left(\mathbf{x}_{n}\right)\right]=\mathbf{k}_{\mathbf{x}}^{T} \mathbf{M} \mathbf{K M k}_{\mathbf{x}}+$ $K_{n n}-2 \mathbf{k}_{\mathbf{x}}^{T} \mathbf{M k}_{\mathbf{x}_{n}}$.

We may translate the feature space Euclidean distance $\tilde{d}^{2}\left[P_{U_{k}} \phi(\mathbf{x}), \phi\left(\mathbf{x}_{n}\right)\right]$ into an equivalent input space Euclidean distance which we may denote $d_{n}^{2}$. Since we use a Gaussian kernel function, we have

$$
\exp \left(-\frac{1}{2 \sigma^{2}} d_{n}^{2}\right)=\frac{1}{2}\left[\|\phi(\mathbf{x})\|^{2}-\tilde{d}^{2}\left[P_{U_{k}} \phi(\mathbf{x}), \phi\left(\mathbf{x}_{n}\right)\right]+\left\|\phi\left(\mathbf{x}_{n}\right)\right\|^{2}\right],
$$

where $\|\phi(\mathbf{x})\|^{2}=\left\|\phi\left(\mathbf{x}_{n}\right)\right\|^{2}=K_{n n}=1$. Hence, $d_{n}^{2}$ is given by

$$
d_{n}^{2}=-2 \sigma^{2} \log \frac{1}{2}\left[2-\tilde{d}^{2}\left[P_{U_{k}} \phi(\mathbf{x}), \phi\left(\mathbf{x}_{n}\right)\right]\right] .
$$

\subsection{The Kwok and Tsang Pre-image Method}

Ideally, the pre-image $\hat{\mathbf{x}}$ should obey the distance constraints $d_{i}^{2}, i=1, \ldots, n$, which may be represented by a column vector $\mathbf{d}^{2}$. However, as pointed out by [4] and others, in general there is no exact pre-image in the input space, so a solution obeying these distance constraints may not exist. Hence, we must settle with an approximation. Using the method in 4, the neighbors are collected in the $(d \times n)$ matrix $\mathbf{X}=\left[\mathbf{x}_{1}, \ldots, \mathbf{x}_{n}\right]$. These are centered at their centroid $\overline{\mathbf{x}}$ by a centering matrix $\mathbf{H}$. Assuming that the training patterns span a $q$-dimensional space, a singular value decomposition is performed $\mathbf{X H}=\mathbf{U} \boldsymbol{\Lambda} \mathbf{V}^{T}=\mathbf{U Z}$, where $\mathbf{Z}=\left[\mathbf{z}_{1}, \ldots, \mathbf{z}_{n}\right]$ is $(q \times n)$ and $\mathbf{d}_{0}^{2}=\left[\left\|\mathbf{z}_{1}\right\|^{2}, \ldots,\left\|\mathbf{z}_{n}\right\|^{2}\right]^{T}$ represents the squared Euclidean distance of each $\mathbf{x}_{n} \in \mathcal{D}_{n}$ to the origin. The Euclidean distance between $\hat{\mathbf{x}}$ and $\mathbf{x}_{n}$ is required to resemble $d_{n}^{2}$ in a least-square sense. The pre-image is then obtained as $\hat{\mathbf{x}}=-\frac{1}{2} \mathbf{U}\left(\mathbf{Z Z}^{T}\right) \mathbf{Z}\left(\mathbf{d}^{2}-\mathbf{d}_{0}^{2}\right)+\overline{\mathbf{x}}$.

\section{Experiments}

We always use $n=7$ neighbors in the experiments. When using centered kernel PCA, we denoise as described in 4 .

Landsat Image. We consider the Landsat multi-spectral satellite image, obtained from 8 . Each pixel is represented by 36 spectral values. Firstly, we extract the classes red soil and cotton yielding a two-class data set. The data is normalized to unit variance in each dimension, since we use a spherical kernel function. 


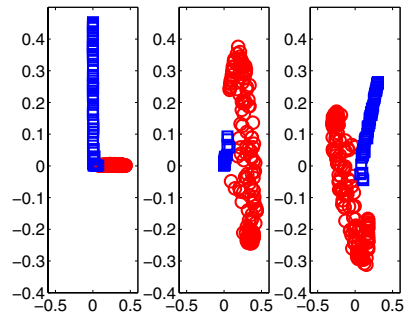

(a) $\sigma=2.8$

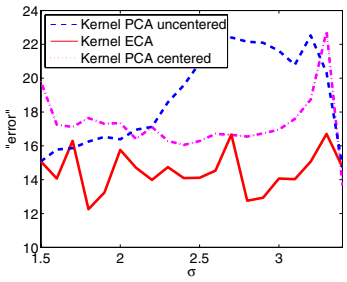

(b)

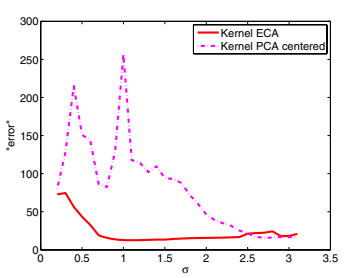

(c)

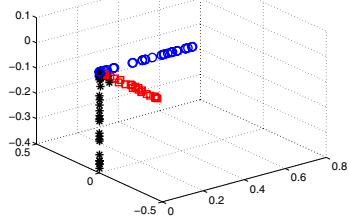

(d) $\sigma=1.5$



(e) $\sigma=1.5$

Fig. 1. Denoising results for the Landsat image, using two and three classes
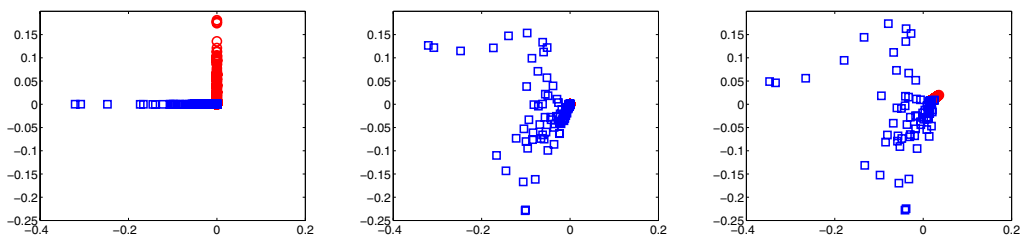

(a) USPS 69 Kernel (b) USPS 69 Kernel (c) USPS 69 Kernel ECA

PCA centered
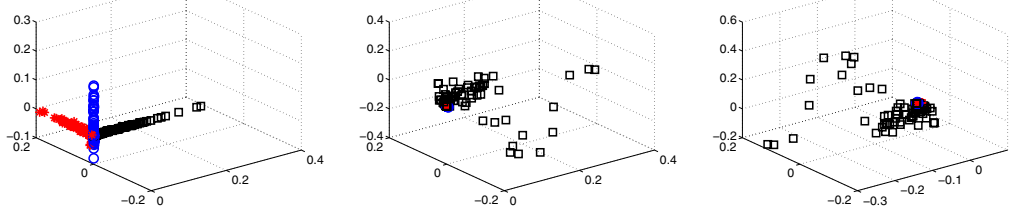

(d) USPS

369 Kernel (e)

(e) USPS 369 Kernel (

(f) USPS 369 Kernel ECA

PCA non-centered

PCA centered

Fig. 2. Examples of Kernel ECA and kernel PCA mapping for USPS handwritten digits

The clean training data is represented by 100 data points drawn randomly from each class. We add Gaussian noise with variance $v^{2}=0.2$ to 50 random test data points ( 25 from each class, not in the training set). Since there are two classes, we 
b6666666669999998999



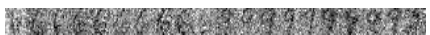

(a) From top: $v^{2}=0.2,0.6,1.5$

66666666669999999999 66666666669999999999 96666666669999999999

(c) KECA, $v^{2}=0.6, k=2,3,10$

99999999999999999999 99999999999999999999 66666666669999999999

(e) $\mathrm{KPCA}, v^{2}=0.2, k=2,3,10$

9898999999999999999 88896898889989988899 2666628289999929299

(g) $\mathrm{KT}, v^{2}=0.2, k=2,3,10$
66666666669999999999 66666666669999999999 66666666669999999999

(b) $\mathrm{KECA}, v^{2}=0.2 k=2,3,10$

66666666669999999999 66666666669999999999 66666666669999999999

(d) KECA, $v^{2}=1.5, k=2,3,10$

99999999999999999999 99999999999999999999 96666666669999999999

(f) $\mathrm{KPCA}, v^{2}=0.6, k=2,3,10$

99999999999999999998

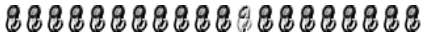

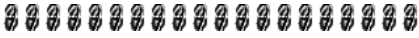

(h) $\mathrm{KT}, v^{2}=0.6, k=2,3,10$

Fig. 3. Denoising of USPS digits 6 and 9

use $k=2$, i.e. two eigenvalues and eigenvectors. For a kernel size $1.6<\sigma<3.3$, $\lambda_{1}, \mathbf{e}_{1}$ and $\lambda_{3}, \mathbf{e}_{3}$ contributes most to the entropy of the training data, and is thus used in kernel ECA. In contrast, kernel PCA always uses the two largest eigenvalues/vectors. Hence kernel ECA and both versions of kernel PCA will denoise differently in this range. In Fig. 1. (a) we illustrate from left to right $\boldsymbol{\Phi}_{e c a}$ and $\boldsymbol{\Phi}_{p c a}$ (using non-centered and centered kernel matrix, respectively.) The kernel size $\sigma=$ 2.8 is used and the classes are marked by different symbols for clarity. Observe how kernel ECA produces a data set with an angular structure, in the sense that each class is distributed radially from the origin, in angular directions which are almost orthogonal. Such a mapping is typical for kernel ECA. The same kind of separation can not be observed for kernel PCA in this case. We quantify the goodness of the denoising of $\mathbf{x}$ by an "error" measure defined as the sum of the elements in $\left|\mathbf{x}^{\prime}-\hat{\mathbf{x}}\right|$, where $\mathbf{x}^{\prime}$ is the clean test pattern and $\hat{\mathbf{x}}$ is the denoised pattern. Fig. 1 (b) displays the mean "error" as a function of $\sigma$ in the range of interest. Of the three methods, kernel ECA is able to denoise with the least error. Secondly, we create a three-class data set by extracting the classes cotton, damp grey soil and vegetation stubble. Fig. 1 (c) shows the denoising error in this case (300 training data, 100 test data) for kernel ECA and centered kernel PCA. Fig. 11(d) and (e) show $\boldsymbol{\Phi}_{e c a}$ and (centered) $\boldsymbol{\Phi}_{p c a}$ for $\sigma=1.5$ (omitting non-centered kernel PCA in this case to save space). Kernel ECA uses $\lambda_{1}, \mathbf{e}_{1}, \lambda_{3}, \mathbf{e}_{3}$ and $\lambda_{4}, \mathbf{e}_{4}$. Also in this case, kernel ECA separates the classes in angular directions. This seems to have a postitive effect on the denoising. 


\section{9 333373363366366666669999999999 333333333366666666669999999999 333333333366666666669999939999 333333333366663666669999999999}

(a) KECA, $\sigma=3.0, \mathrm{k}=3,8,10,15$.

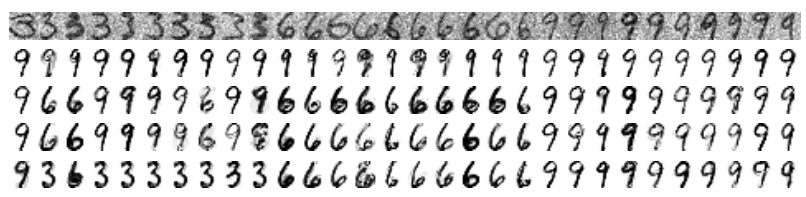

(b) KPCA, $\sigma=3.0, \mathrm{k}=3,8,10,15$.

Fig. 4. Denoising of USPS digits 3, 6 and 9

USPS Handwritten Digits. Denoising experiments are conducted on the $(16 \times$ 16) USPS handwritten digits, obtained from [8], and represented by $(256 \times 1)$ vectors. We concentrate on two and three class problems. In the former case, the data set is composed of the digits six and nine, denoted USPS 69 . In the latter case, we use the digits three, six and nine, denoted USPS 369. For USPS 69 , we use $k=2$, since there are two classes. For $\sigma>3.7$ the the two top kernel ECA eigenvalues are $\lambda_{1}$ and $\lambda_{2}$, which are the same as the two top kernel PCA eigenvalues. Hence, the denoising results will be equal for kernel ECA and non-centered kernel PCA in this case. However, for $\sigma \leq 3.7$, the two top kernel ECA eigenvalues are always different from $\lambda_{1}$ and $\lambda_{2}$, so that kernel ECA and both versions of kernel PCA will be different. As an example, Fig. 2 (a) shows $\boldsymbol{\Phi}_{e c a}$ for $\sigma=2.8$. Here, $\lambda_{1}, \mathbf{e}_{1}$ and $\lambda_{7}, \mathbf{e}_{7}$ is used. Notice also for this data set the typical angular separation provided by kernel ECA. In contrast, Fig. 2 (b) and (c) show non-centered and centered $\boldsymbol{\Phi}_{p c a}$, respectively. Notice how one class (the "nine"s, marked by squares) dominate the other class, especially in (b). Fig. 3 (a) shows ten test digits from each class, with noise added. From top to bottom panel, we have noise variances $v^{2}=0.2,0.6,1.5$. Since there are two classes, we initially perform the denoising using $k=2$. However, we also show results with more dimensions added to the subspace $U_{k}$, for $k=3$ and $k=10$. Fig. 3 (b), (c) and (d) show the kernel ECA results (denoted KECA) for $\sigma=2.8$ for $v^{2}=0.2,0.6,1.5$, respectively. The top panel in each subfigure corresponds to $k=2$, the middle panel corresponds to $k=3$, and in the bottom panel $k=10$ is used. For all noise variances kernel ECA performs very robustly. The results are very good for $k=2$, so the inclusion of more dimensions in the subspace $U_{k}$ does not seem to be necessary. Notice that the shape of the denoised patterns are quite similar. It seems as if the method produces a kind of prototype for each class. This behavior will be further studied below. Fig. 3 (e) and (f) show the non-centered kernel PCA results (denoted KPCA) for $\sigma=2.8$ for $v^{2}=0.2,0.6$, respectively. In both cases, for $k=2$ and $k=3$, the "nine" 


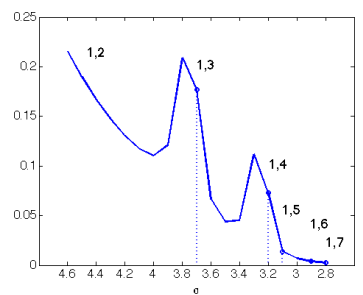

(a)

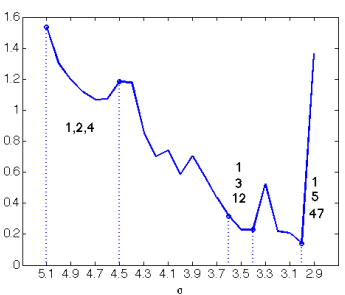

(b)

Fig. 5. Computing the Cauchy-Schwarz class separability criterion as a function of $\sigma$

class totally dominates the "six" class. For each noisy pattern, this means that the nearest neighbors of $P_{U_{k}} \phi(\mathbf{x})$ will always belong to the "nine" class. If we project onto more principal axes, the method improves, as shown in the bottom panel of each figure for $k=10$. Clearly, however, for small subspaces $U_{k}$ kernel ECA performs significantly better than non-centered kernel PCA. Fig. 3 (g) and (h) show the centered kernel PCA results (denoted KT after Kwok and Tsang). In this case, the KT results are much inferior to kernel ECA. Including more principal axes improves the results somewhat, but more dimensions are clearly needed.

When it comes to USPS 369, for $\sigma \leq 5.1$, the three top kernel ECA eigenvalues are always different than $\lambda_{1}, \lambda_{2} \lambda_{3}$, such that kernel ECA and both versions of kernel PCA will be different. For example, for $\sigma=3.0$ kernel ECA uses $\lambda_{1}, \mathbf{e}_{1}, \lambda_{5}, \mathbf{e}_{5}$ and $\lambda_{47}, \mathbf{e}_{47}$, producing a data set with a clear angular structure as shown in Fig. 2 (d). In contrast, Fig. 2 (e) and (f) show non-centered and centered $\boldsymbol{\Phi}_{p c a}$, respectively. The data is not separated as clearly as in kernel ECA. This has an effect on the denoising. Fig. 4 (a) shows the kernel ECA results for $\sigma=3.0$ for $v^{2}=0.2$ and $k=3,8,10,15$ (from top to bottom.) Using only $k=3$, kernel ECA for the most part provides reasonable denoising results, but has some problems distinguishing between the "six" class and the "three" class. In this case, it helps to expand the subspace $U_{k}$ by including a few more dimensions. At $k=8$, for instance, the results are very good by visual inspection. Fig. 4 (b) shows the corresponding non-centered kernel PCA results (centered kernel PCA omitted due to space limitations.) Also in this case, the "nine" class dominates the other two classes. When using $k=15$ principal axes, the results starts to improve, in the sense that all classes are represented. As a final experiment on the USPS 69 and USPS 369 data, we measure the sum of the cosine of the angle between all pairs of class mean vectors of the kernel ECA data set $\boldsymbol{\Phi}_{e c a}$ as a function of $\sigma$. This is equivalent to computing the Cauchy-Schwarz divergence between the class densities as estimated by Parzen windowing [1, and may hence be considered a class separability criterion. We require that the top $k$ entropy components must account for at least $50 \%$ of the total sum of the entropy components, see Eq. (2). Fig. 5 (a) shows the result for USPS 69 using $k=2$. The eigenvalues/vectors used in a region of $\sigma$ are indicated by the numbers above the graph. In this case, the stopping criterion 
is met for $\sigma=2.8$, which yields the smallest value, i,e, the best separation using $\lambda_{1}, \mathbf{e}_{1}$ and $\lambda_{7}, \mathbf{e}_{7}$. Fig. 5 (b) shows the result for USPS 369 using $k=3$. In this case, the best result is obtained for $\sigma=3.0$ using $\lambda_{1}, \mathbf{e}_{1}, \lambda_{5}, \mathbf{e}_{5}$ and $\lambda_{47}, \mathbf{e}_{47}$. These experiments indicate that such a class separability criterion makes sense in kernel ECA, providing the angular structure observed on $\boldsymbol{\Phi}_{e c a}$, and may be developed into a method for selecting an appropriate $\sigma$. This is however an issue which needs further attention in future work.

Finally, we remark that in preliminary experiments not shown here, it appear as if kernel ECA may be a more beneficial alternative to kernel PCA if the number of classes in the data set is relatively low. If there are may classes, more eigenvalues and eigenvectors, or principal components, will be needed by both methods, and as the number of classes grows, the two methods will likely share more and more components.

\section{Conclusions}

Kernel ECA may produce strikingly different spectral data sets than kernel PCA, separating the classes angularly, in terms of the kernel feature space. In this paper, we have exploited this property, by introducing kernel ECA for pattern denoising using the pre-image method proposed in [4]. This requires kernel ECA pre-images to be computed, as derived in this paper. The different behavior of kernel ECA vs. kernel PCA have in our experiments a positive effect on the denoising results, as demonstrated on real data and on toy data.

\section{References}

1. Jenssen, R., Eltoft, T., Girolami, M., Erdogmus, D.: Kernel Maximum Entropy Data Transformation and an Enhanced Spectral Clustering Algorithm. In: Advances in Neural Information Processing Systems 19, pp. 633-640. MIT Press, Cambridge (2007)

2. Schölkopf, B., Smola, A.J., Müller, K.-R.: Nonlinear Component Analysis as a Kernel Eigenvalue Problem. Neural Computation 10, 1299-1319 (1998)

3. Mika, S., Schölkopf, B., Smola, A., Müller, K.R., Scholz, M., Rätsch, G.: Kernel PCA and Denoising in Feature Space. In: Advances in Neural Information Processing Systems, 11, pp. 536-542. MIT Press, Cambridge (1999)

4. Kwok, J.T., Tsang, I.W.: The Pre-Image Problem in Kernel Methods. IEEE Transactions on Neural Networks 15(6), 1517-1525 (2004)

5. Park, J., Kim, J., Kwok, J.T., Tsang, I.W.: SVDD-Based Pattern Denoising. Neural Computation 19, 1919-1938 (2007)

6. Jenssen, R., Erdogmus, D., Principe, J.C., Eltoft, T.: The Laplacian PDF Distance: A Cost Function for Clustering in a Kernel Feature Space. In: Advances in Neural Information Processing Systems 17, pp. 625-632. MIT Press, Cambridge (2005)

7. Shawe-Taylor, J., Cristianini, N.: Kernel Methods for Pattern Analysis. Cambridge University Press, Cambridge (2004)

8. Murphy, R., Ada, D.: UCI Repository of Machine Learning databases. Tech. Rep., Dept. Comput. Sci. Univ. California, Irvine (1994) 